\title{
Canine Dracunculus Nematode Infection, Toledo, Spain
}

\author{
Irina Diekmann, Alaa Aldin Alnassan, Majda Globokar, Nikola Pantchev, Lina Kurzrock, Leticia Hernandez, \\ Javier Lopez, Ricardo Ruano, Silvia Herrero, Georg von Samson-Himmelstjerna, Jürgen Krücken
}

\begin{abstract}
A fragment of a Dracunculus-like worm was extracted from the hind limb of a 2-year-old dog from Toledo, Spain. Cytochrome oxidase I and rRNA sequences confirmed an autochthonous mammalian Dracunculus worm infection in Europe. Sequence analyses suggest close relation to a parasite obtained from a North American opossum.
\end{abstract}

T he nematode genus Dracunculus contains 14 accepted species, 10 of which parasitize reptiles. The mammalian parasites include $D$. fuelleborni, $D$. lutrae, D. insignis and the human pathogen D. medinensis. Adult female Dracunculus worms are located in the host subcutaneous tissue, especially at the distal extremities (1). Human dracunculosis was an important neglected tropical disease, but successful eradication programs have eliminated the parasites from most endemic countries, with the exception of Chad, Ethiopia, South Sudan, Mali, and Angola. In 2019, only 53 human cases but 1,991 animal infections, predominantly in dogs, were reported worldwide (2). Infection occurs by ingestion of copepods containing third-stage larvae through drinking water or by feeding on paratenic hosts such as frogs, tadpoles, or fish $(3,4)$. No genetic differences can be observed between worms infecting humans and dogs, which now appear to be important reservoir hosts (5). In addition, D. lutrae (host: North American river otter Lontra canadensis) and D. insignis (wide host range, including raccoons, several mustelids, and canids) worms infect predominantly carnivores in North America $(6,7)$.

The taxonomy of Dracunculus is based on morphologic characteristics of male worms, which are rarely available. Therefore, identification based on

Author affiliations: Freie Universität Berlin, Berlin, Germany

(I. Diekmann, G. von Samson-Himmelstjerna, J. Krücken); IDEXX

Laboratories, Ludwigsburg, Germany (A.A. Alnassan,

M. Globokar, N. Pantchev, L. Kurzrock); IDEXX Laboratories,

Barcelona, Spain (L. Hernandez, J. Lopez); Clínica Mediterráneo,

Madrid, Spain (R. Ruano, S. Herrero)

DOI: https://doi.org/10.3201/eid2608.201661
$18 \mathrm{~S}$ rRNA has been used for phylogenetic analysis, and identification based on mitochondrial marker cytochrome oxidase C subunit I (COI) has been used for phylogeny, barcoding and intraspecific variation analyses (6).

\section{The Study}

In summer 2018, a 2-year-old dog resembling a podenco (a local breed in Spain) was brought to a veterinarian in Madrid, Spain, with an oozing ulcer laterally in the tarsal region on the left hind limb. The dog had been in the owner's possession for 1 year at the time. Before that, the dog lived on a pig farm in Toledo, Spain, where the previous owner did not take care of the animal and the dog had access to the food and water supplied for the pigs. Because of a case of abuse, the dog was rescued from the farm.

A 12-cm-long front end of a nematode was extracted from the ulcer (Figure 1, panel A). In the preceding year, the dog had been regularly dewormed, wore a collar to protect against fleas and ticks, and was treated with allopurinol against leishmaniosis; the animal had not traveled abroad. After the removal of the nematode fragment by the veterinarian, the nematode was fixed in $70 \%$ ethanol and submitted to IDEXX Laboratories (Ludwigshaven, Germany) for diagnosis. Morphologic examination of the nematode excluded a filarioid species, and a female Dracunculus worm was suspected on the basis of the structure of the papillae around the mouth capsule and the worm size (Figure 1, panel B) (9). For female specimens, species identification is impossible. No larval stages were found in the specimen because only the anterior end of the worm was collected and the uterus was probably lost.

We amplified and sequenced partial nuclear $18 \mathrm{~S}$ and $28 \mathrm{~S}$ rRNA and mitochondrial COI fragments (10-12; Appendix, https://wwwnc.cdc.gov/EID/ article/26/8/20-1661-App1.pdf). From the aligned sequences, we constructed maximum-likelihood phylogenetic trees by using IQtree 1.6.12 (13). Partial 18S rRNA sequences (832 bp, GenBank accession no. MT311138.1) 

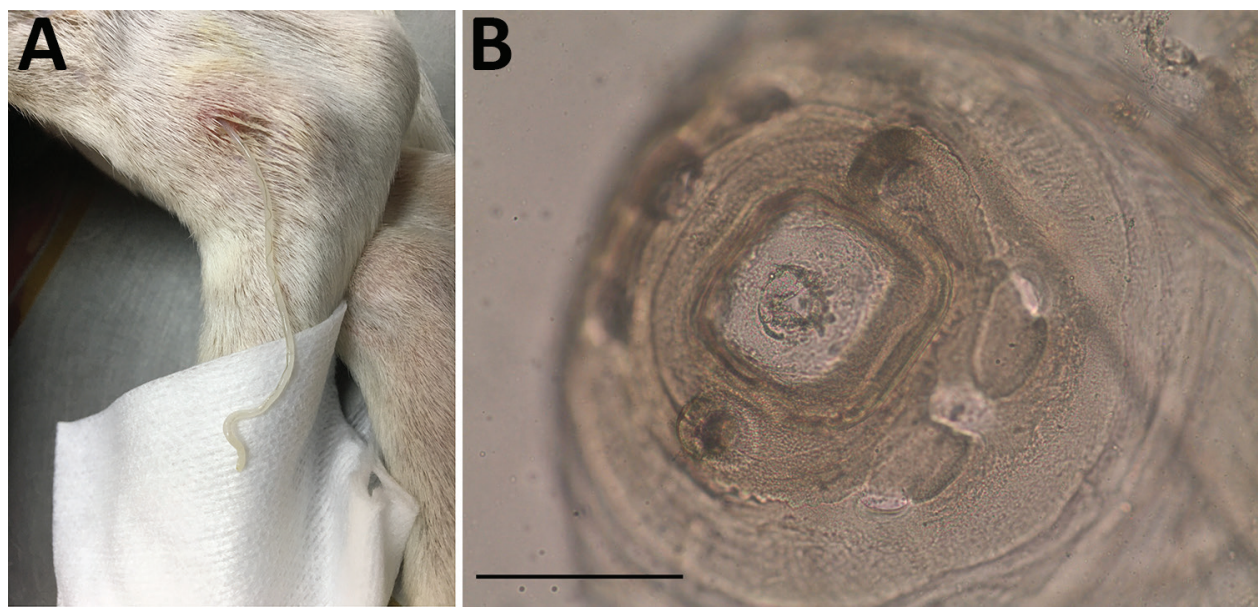

Figure 1. Dracunculus worm extracted from a dog in Toledo, Spain, 2018. A) Nematode extracted from the subcutaneous tissue of tarsal region on the left hind limb of naturally infected dog. B) Microphotograph of the anterior end with characteristic papillae. Scale bar represents $50 \mu \mathrm{m}$. showed $99.6 \%$ identity to D. lutrae (GenBank accession no. JF934737.1), Dracunculus sp. V3104 (GenBank accession no. DQ503457.1), and D. insignis (GenBank accession no. AY947719.1) and $99.5 \%$ identity to D. medinensis (GenBank accession no. MK881307.1). Comparison with D. oesophageus, the only reptile parasite currently listed in GenBank, shared 96.7\% identity (GenBank accession no. AY852269.1).

For the 28S rRNA fragment (231 bp, GenBank accession no. MT311140.1), only 1 homologous sequence from Dracunculus sp. HMM2018 isolate SAN-7590 sequence was available (GenBank accession no. KY990016.1) and showed an identity of 97.8\%. Phylogenetic analysis using $18 \mathrm{~S}$ rRNA data confirmed assignment to the genus Dracunculus and placed the specimen from Spain in a highly supported cluster containing the mammalian parasites $D$. medinensis and $D$. insignis but not the snake parasite D. oesophageus (Appendix Figure). Thus, morphologic and rRNA sequence data agreed in the assignment

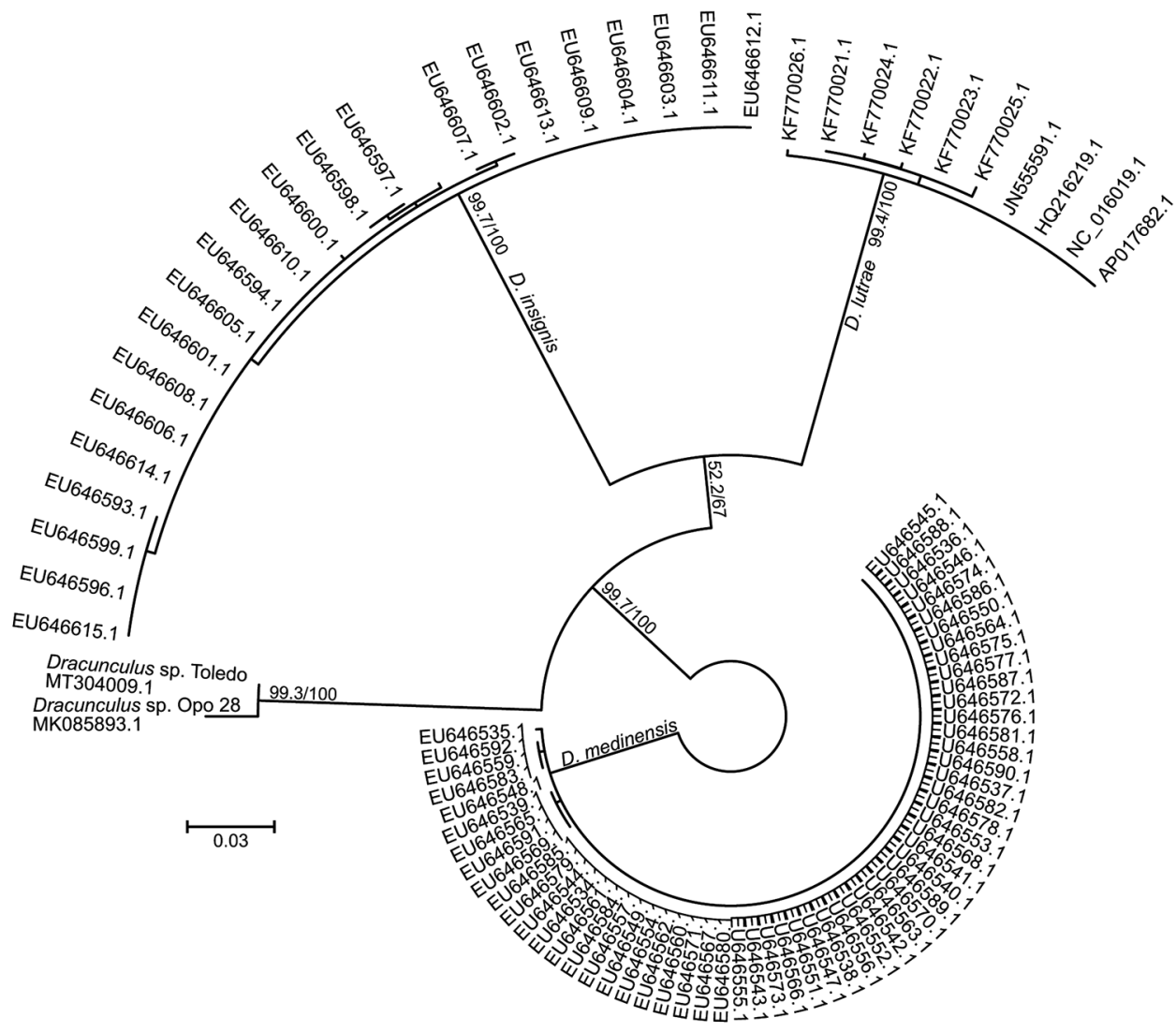

Figure 2. Unrooted maximumlikelihood phylogenetic tree based on Dracunculus spp. mitochondrial marker cytochrome oxidase $\mathrm{C}$ subunit I sequences from a dog in Toledo, Spain, 2018, and reference sequences. Node support values represent results of ultrafast bootstrapping before and the Shimodaira-Hasegawa approximate likelihood ratio test after the slash. Labels at end nodes represent GenBank accession numbers. Scale bar indicates substitutions per site. 
of the specimen to the genus Dracunculus, but no species identification was possible.

The partial COI sequence (654bp, GenBank accession no. MT304009.1) showed that the nematode shared 98.3\% identity with Dracunculus sp. Opo28 (GenBank accession no. MK085893.1), a species found in an opossum (Didelphis virginiana) from Georgia, USA; $92.5 \%$ with D. insignis (GenBank accession no. MK085896.1); 90.7\% with D. lutrae (GenBank accession no. EU646603.1); and 89.3\% identity with D. medinensis (GenBank accession no. AP017682.1). The phylogenetic analysis based on COI sequences positioned the nematode in a cluster together with Dracunculus sp. Opo28, clearly separated from the 3 other Dracunculus species known to infect mammals (Figure 2). The species was designated Dracunculus sp. Toledo.

\section{Conclusions}

Today, the distribution of known Dracunculus worm species infecting mammals is limited to only a few areas endemic for $D$. medinensis worms in Africa, for $D$. lutrae worms in Canada, and for D. insignis worms in North America $(6,7)$. D. fuelleborni worms were identified only once in an opossum in Brazil in 1934 (8). Canine Dracunculus worm infections can be caused by $D$. insignis worms in North America and D. medinensis worms in Africa and Asia. Rarely, canine Dracunculus infections have been reported from nonendemic countries such as Kazakhstan (14); however, because these countries have never been endemic for $D$. medinensis worms, such reports might represent infections with other species. The case in Spain represents an autochthonous mammalian case in Europe, which was considered to be free of such parasites, and manifests a knowledge gap regarding the distribution of Dracunculus spp. worms in mammals $(6,7)$. Although Dracunculus worms had not been reported in mammals in Europe until now, unreported cases might have occurred (e.g., cases in which misidentification resulted in diagnosis of other subcutaneous nematodes, such as Dirofilaria repens).

The close relationship of the worm extracted in Spain to Dracunculus sp. Opo 28 suggests that both specimens belong to the same (or 2 closely related) species. Because Dracunculus sp. Opo 28 was obtained from an opossum, the possibility that both specimens represent the species $D$. fuelleborni cannot be excluded. Given that Dracunculus sp. Opo28 and Dracunculus sp. Toledo worms were found in hosts not closely related to each other as well as on different continents, North America and Europe, the species might have a wide host and geographic range. Opossums are not among the wildlife of Europe, and reservoir hosts for Dracunculus sp. Toledo worms need to be identified. Because Dracunculus sp. Opo28 and Dracunculus sp. Toledo worms are closely related to $D$. insignis, which frequently parasitizes raccoons, importation of this Dracunculus species to Europe with this invasive host species is conceivable and should be further investigated. Although the worm extracted in Spain is not $D$. oesophageus, we cannot exclude entirely the possibility that the nematode is not one of the other reptile parasites that have been described so far only morphologically. Currently, nothing is known about the presence of Dracunculus spp. in copepod intermediate hosts, fish and amphibian paratenic hosts, and mammalian wildlife reservoir hosts in Spain. Because the Dracunculus sp. Toledo worm extracted in Spain had not been reported from dogs previously, canines are probably not epidemiologically relevant hosts. Feeding on infected fish entrails was shown to be a major transmission route to dogs in Chad (15), and infection of the dog in Spain also likely occurred through this route. Obtaining life cycle and prevalence data will require systematic sampling of wildlife and domestic carnivores during necropsies, combined with PCR screening of copepods.

\section{Acknowledgments}

The authors thank the research training group GRK 2046 (Graduierten-Kolleg 2046) for stimulating discussions that substantially improved the manuscript.

I.D. was funded by the Deutsche Forschungsgemeinschaft (German Research Foundation) under project number 251133687/GRK 2046.

\section{About the Author}

Irina Diekmann is a PhD student in the Research Training Group 2046 (Parasite Infections: From Experimental Models to Natural Systems) at the Institute for Parasitology and Tropical Veterinary Medicine, Freie Universität Berlin. Her research interests are molecular characterization of nematodes, parasite life cycles, and parasite-host interactions.

\section{References}

1. Cleveland CA, Garrett KB, Cozad RA, Williams BM, Murray MH, Yabsley MJ. The wild world of Guinea worms: a review of the genus Dracunculus in wildlife. Int J Parasitol Parasites Wildl. 2018;7:289-300. https://doi.org/10.1016/ j.ijppaw.2018.07.002

2. WHO Collaborating Center for Research Training and Eradication of Dracunculiasis, CDC. Guinea worm wrap-up \#265. 2020 [cited 2020 May 4]. https:/ / www.cartercenter. org/resources/pdfs/news/health_publications/ guinea_worm/wrap-up/265.pdf 
3. Cleveland CA, Eberhard ML, Thompson AT, Garrett KB, Swanepoel L, Zirimwabagabo $\mathrm{H}$, et al. A search for tiny dragons (Dracunculus medinensis third-stage larvae) in aquatic animals in Chad, Africa. Sci Rep. 2019;9:375. https://doi.org/10.1038/s41598-018-37567-7

4. Cleveland CA, Eberhard ML, Thompson AT, Smith SJ, Zirimwabagabo $\mathrm{H}$, Bringolf $\mathrm{R}$, et al. Possible role of fish as transport hosts for Dracunculus spp. larvae. Emerg Infect Dis. 2017;23:1590-2. https://doi.org/10.3201/ eid2309.161931

5. Thiele EA, Eberhard ML, Cotton JA, Durrant C, Berg J, Hamm K, et al. Population genetic analysis of Chadian Guinea worms reveals that human and non-human hosts share common parasite populations. PLoS Negl Trop Dis. 2018;12:e006747. https:// doi.org/10.1371/ journal.pntd.0006747

6. Elsasser SC, Floyd R, Hebert PD, Schulte-Hostedde AI. Species identification of North American guinea worms (Nematoda: Dracunculus) with DNA barcoding. Mol Ecol Resour. 2009;9:707-12. https://doi.org/10.1111/ j.1755-0998.2008.02393.x

7. Williams BM, Cleveland CA, Verocai GG, Swanepoel L, Niedringhaus KD, Paras KL, et al. Dracunculus infections in domestic dogs and cats in North America; an underrecognized parasite? Vet Parasitol Reg Stud Rep. 2018;13:148-55. https://doi.org/10.1016/j.vprsr.2018.05.005

8. Travassos L. Dracunculus fuelleborni n. sp., parasito de Didelphis aurita Wied [in Portuguese]. Mem Inst Oswaldo Cruz. 1934;28:235-7. https:/ / doi.org/10.1590/ S0074-02761934000200003

9. Chabaud AG. Spirurida. Camallanoidea, Dracunculoidea, Gnathostomatoidea, Physalopteroidea, Rictularoidea and Thelazioidea. In: Anderson RC, Chabaud AG, Willmott S, editors. Keys to the nematode parasites of vertebrates: archival volume. Wallingford (UK): CABI; 2009. p. 334-60.

10. Blaxter ML, De Ley P, Garey JR, Liu LX, Scheldeman P, Vierstraete A, et al. A molecular evolutionary framework for the phylum Nematoda. Nature. 1998;392:71-5. https:// doi.org/10.1038/32160

11. Demeler J, Ramünke S, Wolken S, Ianiello D, Rinaldi L, Gahutu JB, et al. Discrimination of gastrointestinal nematode eggs from crude fecal egg preparations by inhibitor-resistant conventional and real-time PCR. PLoS One. 2013;8:e61285. https://doi.org/10.1371/journal.pone.0061285

12. Duscher GG, Harl J, Fuehrer HP. Evidence of Troglotrema acutum and Skrjabingylus sp coinfection in a polecat from Lower Austria. Helminthologia. 2015;52:63-6. https://doi.org/10.1515/helmin-2015-0011

13. Trifinopoulos J, Nguyen LT, von Haeseler A, Minh BQ. W-IQ-TREE: a fast online phylogenetic tool for maximum likelihood analysis. Nucleic Acids Res. 2016;44(W1):W232-5. https://doi.org/10.1093/nar/gkw256

14. Chun-Syun F. Dracunculus infection in dogs in Kazakhstan [in Russian]. Med Parazitol (Mosk). 1958;27:219-20.

15. McDonald RA, Wilson-Aggarwal JK, Swan GJF, Goodwin CED, Moundai T, Sankara D, et al. Ecology of domestic dogs Canis familiaris as an emerging reservoir of Guinea worm Dracunculus medinensis infection. PLoS Negl Trop Dis. 2020;14:e0008170. https:// doi.org/10.1371/ journal.pntd.0008170

Address for correspondence: Jürgen Krücken, Freie Universität Berlin, Institute for Parasitology and Tropical Veterinary Medicine, Robert-von-Ostertag Str. 7-13, 14163 Berlin, Germany; email: juergen.kruecken@fu-berlin.de

\section{EID Podcast: Epidemiology of Human Plague in the United States, 1900-2012}

Plague is a globally distributed, zoonotic disease caused by the bacterium Yersinia pestis. In the late 1890 s, rat-infested steamships introduced the disease into the continental United States. The first documented autochthonous human infection occurred in the Chinatown section of San Francisco, California, in March of 1900. Cases were soon reported in other port cities, including New Orleans, Galveston, Seattle, and Los Angeles. Along the Pacific Coast, infection spread from urban rats to native rodent species, and by the 1950s, Y. pestis had spread eastward to reach western portions of the Dakotas, Nebraska, Kansas, Oklahoma, and Texas. This distribution has remained static for more than 60 years, presumably the result of climatic and ecologic factors that limit further spread. Although poorly defined, these factors may be related to the ecology of vector species rather than that of rodent hosts.

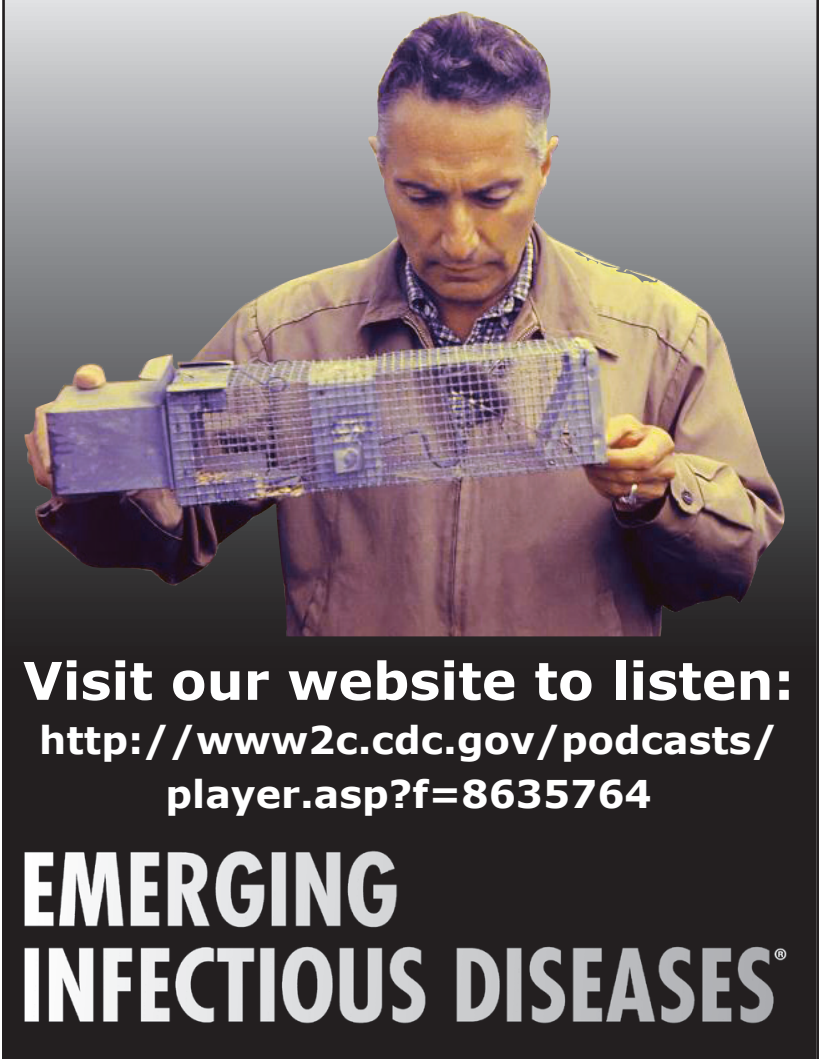

